Georgian Mathematical Journal

Volume 14 (2007), Number 4, 607-626

\title{
ON WIENER'S CRITERION FOR AN ELLIPTIC EQUATION WITH NONUNIFORM DEGENERATION
}

\author{
RABIL A. AMANOV AND FARMAN I. MAMEDOV
}

\begin{abstract}
For some class of nonuniformly degenerated elliptic equations of second order, a necessary and sufficient condition for boundary points to be regular is found. This condition is an analogue of Wiener's criterion for the Laplace equation.
\end{abstract}

2000 Mathematics Subject Classification: 35B65, 35J67.

Key words and phrases: Wiener's criterion, boundary regularity conditions, elliptic equations, nonuniformly degenerated.

\section{INTRODUCTION}

Let $E_{n}$ be an $n$-dimensional space of points $x=\left(x_{1}, x_{2}, \ldots, x_{n}\right), n \geq 2, D$ be the bounded domain in $E_{n}$ with boundary $\partial D, 0 \in \partial D$. We will consider the equation

$$
L u=\sum_{i, j=1}^{n} \frac{\partial}{\partial x_{i}}\left(a_{i j}(x) \frac{\partial u}{\partial x_{j}}\right)=0
$$

in the domain $D$. The elements of the matrix $A=\left\|a_{i j}(x)\right\|$ given in $E_{n}$ are symmetrical, real, measurable and satisfy the condition

$$
\mu \sum_{j=1}^{n} \rho(x)^{\alpha_{j}} \eta_{j}^{2} \leq \eta \cdot A \eta \leq \mu^{-1} \sum_{j=1}^{n} \rho(x)^{\alpha_{j}} \eta_{j}^{2},
$$

where $\eta$ is an arbitrary vector in $E_{n}, \mu=$ const $\leq 1$. Here and in what follows the point denotes a usual scalar product in $E_{n}, \rho(x)=\max _{1 \leq i \leq n}\left|x_{i}\right|^{1 / \sigma_{i}}$. With regard to the constants we also make the following assumptions:

$$
\sigma_{i}=\frac{\alpha_{i}+\beta}{2}>0, \quad\left|\alpha_{i}\right|<\sum_{j=1}^{n} \sigma_{j}, \quad \beta \geq 0,
$$

where $i=1,2, \ldots, n$.

The aim of this paper is to prove a Wiener type criterion of the regularity of a boundary point (see [1] and [2]) in the case of the first boundary value problem for equation (1.1), which admits nonuniform power degeneration at the point 0 . For equation (1.1) without degeneration $\left(\alpha_{i}=0, i=1,2, \ldots, n\right)$, the criterion of regularity of boundary points was obtained in the fundamental work [3] (for another proof see [4]). The corresponding results for nondivergent equations with Dini continuous coefficients (or with a ellipticity function) can be found 
in [5], [6] ([7]). With regard to equation (1.1) we also want to mention the important work [8] in the case of uniform degeneration of $\omega(x)$ (which replaces all $\rho(x)^{\alpha_{i}}, i=1,2, \ldots, n$, in condition (1.2)) satisfying the Muckenhoupt condition $A_{2}$.

Note that in the case of equation (1.1) the question of internal regularity satisfying condition (1.2) is considered in [9]-[11].

\section{Definitions, the Notation and Auxiliary Statements}

We denote by $\nabla u=\left(u_{x_{1}}, u_{x_{2}}, \ldots, u_{x_{n}}\right)$ the gradient of the function $u(x)$; $\rho^{\alpha} \nabla u$ is the vector $\left(\rho(x)^{\alpha_{1}} u_{x_{1}}, \rho(x)^{\alpha_{2}} u_{x_{2}}, \ldots, \rho(x)^{\alpha_{n}} u_{x_{n}}\right) . \quad\left\|\rho(x)^{\alpha / 2} \nabla u\right\|_{2, D}$ stands for the sum $\sum_{j=1}^{n}\left\|\rho(x)^{\alpha_{j} / 2} \nabla u_{x_{j}}\right\|_{2, D}$, where $\|\cdot\|_{2, D}$ is a usual Lebesgue norm in $L_{2}(D)$.

We denote by $\operatorname{Lip}(D)$ the space of Lipshitz continuous functions having a continuous extension in $\bar{D}$, by $\operatorname{Lip}_{0}(D)$ a subspace of functions $\operatorname{Lip}(D)$ vanishing on $\partial D$. $W_{2, \alpha}^{1}(D)$ is the closure of the space $\operatorname{Lip}(D)$ with respect to the norm

$$
\|f\|=\|f\|_{2, D}+\left\|\rho^{\alpha / 2} \nabla u\right\|_{2, D}
$$

where $\|f\|_{2, D}=\|f\|_{L_{2}(D)} \cdot \stackrel{\circ}{W}_{2, \alpha}^{1}(D)$ is a subspace of $W_{2, \alpha}^{1}(D)$, in which the set of all functions $\operatorname{Lip}_{0}(D)$ is dense.

We denote by $Q_{R}^{x}$ a quasiball $\left\{y \in E_{n}: \rho(y-x)<R\right\}$.

Definition 2.1. We say that a function $u(x) \in W_{2, \alpha}^{1}(D)$ is not greater (smaller) than the constant $M$ on a set $E \subset \bar{D}$ in the sense of $W_{2, \alpha}^{1}(D)$ if there exists a sequence of functions $u_{j} \in \operatorname{Lip}(D)$ such that 1) $u_{j}(x) \leq M\left(u_{j}(x) \geq M\right)$ on $E$ and 2) $\left\|u_{j}-u\right\| \rightarrow 0$ in the norm of the space $W_{2, \alpha}^{1}(D)$ as $j \rightarrow \infty$.

Definition 2.2. Any sequence $u_{j}(x) \in \operatorname{Lip}(D)$ satisfying condition 2) is called approximating for a function $u(x) \in W_{2, \alpha}^{1}(D)$.

Definition 2.3. A function $u(x) \in W_{2, \alpha}^{1}(D)$ is said to be equal to $M$ on a set $E \subset \bar{D}$ in the sense of $W_{2, \alpha}^{1}(D)$ if simultaneously $u(x) \geq M$ and $u(x) \leq M$ on $E$ in the sense of $W_{2, \alpha}^{1}(D)$.

Note that a function $u(x) \in W_{2, \alpha}^{1}(D)$ is equal to $M$ if and only if there exists an approximating sequence $u_{j}(x)$ equal to $M$ on the set $E$.

Let $k \in R, u(x) \in W_{2, \alpha}^{1}(D)$. Denote by $\{u\}_{k}=\max (u(x), k),\{u\}^{k}=$ $\min (u(x), k)$ the sections of the function $u(x)$.

Lemma 2.1. A section $\{u\}_{k}$ of a function $u(x) \in W_{2, \alpha}^{1}(D)$ belongs to this space and, if $u_{j}$ approximates $u(x)$, then sections $\left\{u_{j}\right\}_{k}$ will approximate $\{u\}_{k}$, $\left\|\left\{u_{j}\right\}_{k}-\{u\}_{k}\right\| \rightarrow 0$ in the norm $W_{2, \alpha}^{1}(D)$ as $j \rightarrow \infty$.

Proof. We extract from $u_{j}$ an a.e. converging subsequence. Preserving for it the notation of the original sequence, we have

$$
u_{j} \rightarrow u, \quad \nabla u_{j} \rightarrow \nabla u \quad \text { a.e. } \quad x \in D \quad \text { as } j \rightarrow \infty \text {. }
$$


It is obvious that $\left\{u_{j}\right\}_{k} \in \operatorname{Lip}(D),\{u\}_{k}=k+(u-k) \chi_{u>k},\left\{u_{j}\right\}_{k}=k+\left(u_{j}-\right.$ $k) \chi_{u_{j}>k}$, where $\chi_{E}$ denotes the characteristic function of the set $E$. Therefore

$$
\left\{u_{j}\right\}_{k}-\{u\}_{k}=\left(u_{j}-u\right) \chi_{u_{j}>k}+(u-k)\left(\chi_{u_{j}>k}-\chi_{u>k}\right) .
$$

By the Minkovski inequality, from (2.2) we have

$$
\left\|\left\{u_{j}\right\}_{k}-\{u\}_{k}\right\|_{2, D} \leq\left\|\left(u_{j}-u\right) \chi_{u_{j}>k}\right\|_{2, D}+\left\|(u-k)\left(\chi_{u_{j}>k}-\chi_{u>k}\right)\right\|_{2, D} \rightarrow 0
$$

as $j \rightarrow \infty$. Indeed, the first summand does not exceed $\left\|u_{j}-u\right\|_{2, D}$ which tends to zero, while the second summand tends to zero by virtue of Lebesgue's majorant theorem, since $\chi_{u_{j}>k} \rightarrow \chi_{u>k}$ a.e. for almost all $x \in D$. Furthermore, (2.2) readily implies that

$$
\nabla\left\{u_{j}\right\}_{k}-\nabla\{u\}_{k}=\nabla\left(u_{j}-u\right) \chi_{u_{j}>k}+\nabla u\left(\chi_{u_{j}>k}-\chi_{u>k}\right) .
$$

Then

$$
\begin{aligned}
\left\|\rho^{\alpha / 2} \nabla\left(\left\{u_{j}\right\}_{k}-\{u\}_{k}\right)\right\|_{2, D} \leq & \left\|\chi_{u_{j}>k} \cdot \rho^{\alpha / 2} \nabla\left(u_{j}-u\right)\right\|_{2, D} \\
& +\left\|\left(\chi_{u_{j}>k}-\chi_{u>k}\right) \rho^{\alpha / 2} \nabla u\right\|_{2, D} \rightarrow 0,
\end{aligned}
$$

also by virtue of Lebesgue's theorem and the fact that $u_{j}(x)$ approximates $u(x)$.

We have proved that

$$
\left\|\left\{u_{j}\right\}_{k}-\{u\}_{k}\right\|_{2, D} \rightarrow 0 \quad \text { as } \quad j \rightarrow \infty
$$

with respect to the norm $W_{2, \alpha}^{1}(D)$ for some subsequence $u_{j}$. If this relation is violated for some subsequence $u_{j_{n}}$, then, applying the above-given arguments to this subsequence, we come to a contradiction.

Note that in case of Sobolev space $W_{p}^{1}(D)$ the analogous statements in $[12$, Ch. 2, §3] and also [3, Lemma 1.1] are proved using other approaches.

Definition 2.4. A function $u(x) \in W_{2, \alpha}^{1}(D)$ is called a solution of equation (1.1) in the domain $D$ if

$$
\int_{D} \nabla u \cdot A(x) \nabla \varphi d x=0, \quad \forall \varphi \in \operatorname{Lip}_{0}(D) .
$$

It is not difficult to verify that if $u(x) \in W_{2, \alpha}^{1}(D)$ is a solution of equation (1.1), then identity (2.3) is fulfilled for any test function $\varphi \in \stackrel{\circ}{W_{2, \alpha}^{1}}(D)$.

Definition 2.5. A function $u \in W_{2, \alpha}^{1}(D)$ satisfying the inequality

$$
\int_{D} \nabla u \cdot A(x) \nabla \varphi d x \underset{(\geq)}{\leq} 0
$$

for any $\varphi \in \operatorname{Lip}_{0}(D), \varphi \geq 0$, is called a subsolution (supersolution) of the operator $L$ in $D$. 
Definition 2.6. Let $H \subset E_{n}$ be a compact subset. We say that the capacity of the compactum $H$ is the number

$$
\operatorname{cap}_{\alpha} H=\inf \int_{E_{n}} \rho^{\alpha}(x) \nabla u \cdot \nabla u d x,
$$

where the lower bound is taken over all admissible functions $u \in \operatorname{Lip}_{0}\left(E_{n}\right)$, $u(x) \geq 1$ on $H$.

The next lemma is an analogue of the corresponding statement in [13].

Lemma 2.2 (Maximum Principle). Let $u(x) \in W_{2, \alpha}^{1}(D)$ be a solution of equation (1.1) in the domain $D$ and $u(x) \leq M(u(x) \geq M)$ on the boundary $\partial D$ of the domain $D$ in the sense of $W_{2, \alpha}^{1}(D)$. Then $u(x) \leq M(u(x) \geq M)$ for almost all $x \in D$.

Proof. Let $\varepsilon>0$ be an arbitrary integer. By virtue of Lemma 2.1 the function $\varphi=\{u\}_{M+\varepsilon}-(M+\varepsilon)$ belongs to $\stackrel{\circ}{W}_{2, \alpha}^{1}(D)$. Substituting this test function into identity (2.3), we have

$$
\int_{D} \nabla\{u\}_{M+\varepsilon} \cdot A \nabla\{u\}_{M+\varepsilon}=0,
$$

from which, using the Sobolev-Gagliardo inequality and conditions (1.2), (1.3) we obtain

$$
\begin{aligned}
\|\{u\}_{M+\varepsilon} & -(M+\varepsilon)\left\|_{\frac{n}{n-1}, D} \leq C\right\| \nabla\{u\}_{M+\varepsilon} \|_{1, D} \\
& \leq C\left(\int_{D} \sum_{j=1}^{n} \rho^{\alpha_{j}}\left|\nabla\{u\}_{M+\varepsilon}\right|^{2} d x\right)^{1 / 2}\left(\int_{D} \sum_{j=1}^{n} \rho^{-\alpha_{j}}(x) d x\right)^{1 / 2} \\
& \leq C \int_{D} \nabla\{u\}_{M+\varepsilon} \cdot A \nabla\{u\}_{M+\varepsilon} d x=0, \quad C=C(n, \mu, \alpha, D) .
\end{aligned}
$$

Then $\{u\}_{M+\varepsilon} \leq M+\varepsilon$ a.e. for almost all $x \in D$ and taking into account the arbitrariness of $\varepsilon>0$, we find that $u(x) \leq M$ for almost all $x \in D$.

\section{Capacity and the Potential}

Let $H \subset Q$ be a compact subset of the quasiball $Q$. Following $[3, \S 4]$, let us consider the variational problem

$$
D(v)=\int_{Q} \nabla v \cdot A \nabla v d x \rightarrow \inf
$$

in the class $S$ of admissible functions, $v \in \operatorname{Lip}_{0}(Q), v(x) \geq 1$ on $H$. The set $S \subset W_{2, \alpha}^{1}(Q)$ is convex, but not closed. Denote by $\bar{S}$ the closure of $S$. Since the functional $D(v)$ is continuous, the lower bound $D(v)$ on $\bar{S}$ coincides with 
the lower bound on $S$. The functional is strongly convex in $W_{2, \alpha}^{1}(Q)$ : for any $u, v \in W_{2, \alpha}^{1}(Q)$ we have

$$
D\left(\frac{u+v}{2}\right)-\frac{1}{2} D(u)-\frac{1}{2} D(v)=-\frac{1}{4} D(u-v) \leq-\frac{\mu}{4}\|u-v\|_{W_{2, \alpha}^{1}(Q)}^{2} .
$$

Since the set $\bar{S}$ is convex and closed, there exists a function $v_{H}$ such that the functional $D(v)$ attains its lower bound on $\bar{S}$. We will show that $v_{H}$ is unique. Let inf $D(f)=D(u)=D(v)=d$ simultaneously for two different functions $u, v \in W_{2, \alpha}^{1}(D)$. If $u=t v, t \in R$, then we have $d=D(v)=D(t v)=t^{2} D(v)=$ $t^{2} d$, whence $t=1$, i.e. $u=v$. If $u, v$ are linearly independent, then the strict inequality

$$
\| \int_{Q} \nabla v \cdot A \nabla u d x \mid<\sqrt{D(u) \cdot D(v)}
$$

is valid. Therefore for $\frac{u+v}{2} \in \bar{S}$ we have

$$
D\left(\frac{u+v}{2}\right)=\frac{1}{4} D(u)+\frac{1}{4} D(v)+\frac{1}{2} \int_{Q} \nabla u \cdot A \nabla v d x<d,
$$

which contradicts the condition $D(u)=$ inf.

Definition 3.1. Let $H \subset Q$ be a compactum, $v_{H} \in \stackrel{\circ}{W}_{2, \alpha}^{1}(Q)$ be a unique solution of the variational problem

$$
D(f) \rightarrow \inf , \quad f \in \bar{S} .
$$

Then the function $v_{H}$ is called the potential of the set $H$.

If $\left\{v_{H}\right\}^{1}$ is a section of the function $v_{H}$, then by virtue of Lemma 2.1 we have $\left\{v_{H}\right\}^{1} \in \bar{S}$ and therefore $D\left(\left\{v_{H}\right\}^{1}\right) \leq D\left(v_{H}\right)=$ inf. Thus $v_{H}=1$ on $H$ in the sense of $\stackrel{\circ}{W}_{2, \alpha}^{1}(Q)$. For any $\varepsilon>0, \psi \in \operatorname{Lip}_{0}(Q), \psi \geq 0$ we have

$$
D\left(v_{H}+\varepsilon \psi\right) \geq D\left(v_{H}\right) \text { on } H,
$$

whence, applying the well known variational technique, we obtain

$$
\int_{Q} \nabla v_{H} \cdot A \nabla \psi d x \geq 0
$$

i.e. $v_{H}$ is a supersolution of the operator $L$ in $Q$. If in (3.3) we take an arbitrary $\varepsilon$, for any $\psi \in \operatorname{Lip}_{0}(Q \backslash H)$, then for $v_{H}$ we obtain the identity $\int_{Q} \nabla v_{H} \cdot A \nabla \psi d x=$ 0, i.e. $v_{H}$ is a solution of equation (1.1) outside $H$. Finally, applying the maximum principle to the potential $v_{H}$, we obtain $0 \leq v_{H} \leq 1$. Thus we have proved the following statement.

Lemma 3.1. Let $H \subset Q$ be a compact subset. Then there exists a unique solution of problem (3.2) with the properties: the function $v_{H}$ delivers inf to the functional $D(f)$ and is a unique function in this sense; the function $v_{H}$ is a 
supersolution of the operator $L$ in $Q ; v_{H}$ is a solution of equation (1.1) outside $H$; $v_{H}$ satisfies the inequality $0 \leq v_{H}(x) \leq 1$ a.e. in $Q ; v_{H}=1$ for $x \in \operatorname{int} H$.

Now let us consider the operator

$$
L_{1} v=\sum_{i=1}^{n} \frac{\partial}{\partial x_{i}}\left(\rho(x)^{\alpha_{i}} \frac{\partial v}{\partial x_{i}}\right) .
$$

All that has been said above refers to the functional

$$
D_{1}(v)=\int_{Q} \rho(x)^{\alpha} \nabla v \cdot \nabla v d x
$$

too, i.e. the following statement is true.

Lemma 3.2. For any compactum $H \subset Q$, the variational problem

$$
D_{1}(v) \rightarrow \inf , \quad v \in \bar{S}
$$

has a unique solution $v_{H}$. The number

$$
\overline{\operatorname{cap}}_{\alpha} H=D_{1}\left(v_{H}\right)=\inf
$$

is called the capacity of the compactum $H$ with respect to the quasiball $Q$ (if $Q$ coincides with $E_{n}$, then we have the capacity from Definition 2.6). The function $v_{H}$ is a solution of the equation $L_{1} u=0$ in $Q \backslash H$, a supersolution of the operator $L_{1}$ in $Q$, and also $0 \leq v_{H} \leq 1, v_{H}=1$ on $H$ in the sense of $W_{2, \alpha}^{1}(D)$.

The next two lemmas are the obvious formulas for a classical solution of problem (1.1).

Lemma 3.3. Let $H \subset E_{n}$ be a compact subset, $v \in \stackrel{\circ}{W}_{2, \alpha}^{1}\left(E_{n}\right)$ be its potential, and $v^{(k)}(x)$ approximate $v(x)$ as $k \rightarrow \infty, G=E_{n} \backslash H$. Then for almost all $t \in(0,1)$ we have the identity

$$
t \int_{\partial \Gamma_{t}} \nabla v^{(k)} \cdot A N d \sigma=\int_{E_{n} \backslash \Gamma_{t}} \nabla v^{(k)} \cdot A \nabla v^{(k)} d x+\delta_{k},
$$

where $\delta_{k} \rightarrow 0$ as $k \rightarrow \infty, N=\left(N_{1}, N_{2}, \ldots, N_{m}\right)$ is the inward normal vector to the domain boundary

$$
\Gamma_{t}=\left\{x \in E_{n}: v^{(k)}(x)>t\right\}
$$

(depending also on $k$ ).

Proof. Let $\varphi=g_{h}(x) v^{(k)}(x)$, where $g_{h}(x)=\frac{t-v^{(k)}(x)}{h}$ for $x \in \Gamma_{t-h} \backslash \Gamma_{t}, g_{h}(x)=1$ for $x \in E_{n} \backslash \Gamma_{t-h}, g_{h}(x)=0$ for $x \in \Gamma_{t}, t \in(0,1], h$ is sufficiently small. It is obvious that the function $\varphi \in \stackrel{\circ}{W}_{2, \alpha}^{1}(G)$, and since from the identity

$$
\int_{G} \nabla v \cdot A \nabla \varphi d x=0
$$


it follows that

$$
\int_{G} \nabla v^{(k)} \cdot A \nabla \varphi d x=\delta_{k} \rightarrow 0 \quad \text { as } \quad k \rightarrow \infty
$$

after substituting the expression for $\varphi$ into this identity we obtain

$$
-\frac{1}{h} \int_{\Gamma_{t-h} \backslash \Gamma_{t}} v^{(k)} \nabla v^{(k)} \cdot A \nabla v^{(k)} d x+\int_{E_{n} \backslash \Gamma_{t}} g_{h} \nabla v^{(k)} \cdot A \nabla v^{(k)} d x=\delta_{k} .
$$

Using Federer's formula [14, p. 40] and Lebesgue's theorem [15, §1, Ch. 1] for almost all $t \in(0,1)$, we obtain for the first summand in (3.10)

$$
\begin{array}{r}
\frac{1}{h} \int_{\Gamma_{t-h} \backslash \Gamma_{t}} v^{(k)} \nabla v^{(k)} \cdot A \nabla v^{(k)} d x=\frac{1}{h} \int_{t-h}^{t} s d s\left(\int_{\partial \Gamma_{s}} N \cdot A \nabla v^{(k)} d \sigma_{s}\right) \\
\rightarrow t \cdot \int_{\partial \Gamma_{t}} \nabla v^{(k)} \cdot A N d \sigma_{t}
\end{array}
$$

as $h \rightarrow 0$, since $N=\nabla v^{(k)} /\left|\nabla v^{(k)}\right|$ on the surface $\partial \Gamma_{s}$. By virtue of Lebesgue's majorant theorem we have

$$
\int_{E_{n} \backslash \Gamma_{t}} g_{h} \nabla v^{(k)} \cdot A \nabla v^{(k)} d x \rightarrow \int_{E_{n} \backslash \Gamma_{t}} \nabla v^{(k)} \cdot A \nabla v^{(k)} d x \quad \text { as } \quad h \rightarrow 0 .
$$

Passing to the limit as $h \rightarrow 0$ and taking into account (3.11), (3.12) we obtain (3.8).

Lemma 3.4. Let $H \subset E_{n}$ be a compactum, $v(x), v^{(k)}(x), \Gamma_{t}$ be the same as in Lemma 3.3. Then for a.e. for almost all $0<s<t<1$ the identity

$$
\int_{\partial \Gamma_{s}} \nabla v^{(k)} \cdot A N d \sigma_{s}=t \cdot \int_{\partial \Gamma_{t}} \nabla v^{(k)} \cdot A N d \sigma_{t}+\delta_{k}
$$

is valid, where $\delta_{k} \rightarrow 0$ as $k \rightarrow \infty, d \sigma_{s}$ is an element of the $(n-1)$-dimensional Lebesgue measure on the surface $\partial \Gamma_{s}$ (depends on $k$ ).

The proof of this lemma is analogous to that of Lemma 3.3. In identity (3.9) we set the test function $\varphi=g_{h}(x) f_{h}(x)$, where $g_{h}(x)$ is the same as in Lemma 3.3, $f_{h}(x)=\frac{v^{(k)}(x)-s}{h}$ for $x \in \Gamma_{s} \backslash \Gamma_{s+h}, f_{h}(x)=1$ for $x \in \Gamma_{s+h}, f_{h}(x)=0$ for $x \in E_{n} \backslash \Gamma_{s}$. Then

$$
-\frac{1}{h} \int_{\Gamma_{s} \backslash \Gamma_{s+h}} v^{(k)} \nabla v^{(k)} \cdot A \nabla v^{(k)} d x+\frac{1}{h} \int_{\Gamma_{t-h} \backslash \Gamma_{t}} v^{(k)} \nabla v^{(k)} \cdot A \nabla v^{(k)} d x=\delta_{k} \rightarrow 0
$$

as $k \rightarrow \infty$. If $h \rightarrow 0$, then analogously to (3.11) we obtain (3.13). 


\section{The Harnack Inequality}

Theorem 4.1. Let $G_{R}=Q_{R}^{0} \backslash Q_{R / 4}^{0}, u(x) \in W_{2, \alpha}^{1}\left(G_{R}\right)$ be a positive solution of equation (1.1) in $G_{R}$. Then there exists a constant $C=C(n, \mu, \alpha, \beta)$ such that

$$
\underset{\partial Q_{r / 2}^{0}}{\operatorname{ess} \sup } u(x) \leq C \underset{\partial Q_{r / 2}^{0}}{\operatorname{essinf}} u(x) .
$$

Proof. By definition, for any $\varphi \in W_{2, \alpha}^{1}\left(G_{R}\right)$

$$
\int_{G_{R}} \nabla v \cdot A \nabla \varphi d x=0 .
$$

Performing the change $x=R^{\sigma} \xi=\left(R^{\sigma_{1}} \xi_{1}, R^{\sigma_{2}} \xi_{2}, \ldots, R^{\sigma_{n}} \xi_{n}\right)$, we obtain

$$
\int_{T} \nabla_{\xi} v \cdot B \nabla_{\xi} \psi d \xi=0
$$

where $T=Q_{1}^{0} \backslash Q_{1 / 4}^{0}$ is the image of the set $G_{R} ; B=\left\|b_{i j}(\xi)\right\|, b_{i j}=R^{\beta-\sigma_{i}-\sigma_{j}} \times$ $a_{i j}(x(\xi)), i, j=1,2, \ldots, n . x(\xi)=R^{\sigma} \xi, \nabla_{\xi} v$ denotes the vector $\left(v_{\xi_{1}}, v_{\xi_{2}}, \ldots, v_{\xi_{n}}\right)$, and $\psi(\xi)=\varphi(x(\xi))$ is an arbitrary function from the space $\stackrel{\circ}{W}_{2}^{1}(T), v(\xi)=$ $u(x(\xi))$.

The matrix $B$ is uniformly elliptic on $T$. Indeed,

$$
\mu \sum_{j=1}^{n} \rho(x(\xi))^{\alpha_{j}} R^{\beta-2 \sigma_{j}} \eta_{j}^{2} \leq \eta \cdot B \eta \leq \mu^{-1} \sum_{j=1}^{n} \rho(x(\xi))^{\alpha_{j}} R^{\beta-2 \sigma_{j}} \eta_{j}^{2} .
$$

Since $x \in G_{R}$ for $\xi \in T$, we have

$$
\frac{R}{4}<\rho(x)<R
$$

and therefore

$$
|\eta|^{2} \frac{\mu}{4^{\alpha_{0}}} \leq \eta \cdot B \eta \leq \mu^{-1} 4^{\alpha_{0}}|\eta|^{2} \quad \forall \eta \in E_{n}
$$

where $\alpha_{0}=\max _{1 \leq j \leq n}\left|\alpha_{j}\right|$. The image of the points $x \in \partial Q_{r / 2}^{0}$ belongs to $T^{\prime}=\partial Q_{1 / 2}^{0}$.

It is obvious that $T^{\prime} \subset T$ and a usual distance $\operatorname{dist}\left(T^{\prime}, \partial T\right)>0$ does not depend on $R$. The function $v(\xi)$ belongs to $W_{2}^{1}(T)$, since $u(x) \in W_{2, \alpha}^{1}\left(G_{R}\right)$. Indeed, from (4.4) we have

$$
\int_{G_{R}} \rho^{\alpha} \nabla u \cdot \nabla u d x=\sum_{j=1}^{n} R^{\sum_{i=1}^{n} \sigma_{i}} \int_{T} \rho(x(\xi))^{\alpha_{j}} R^{-2 \sigma_{j}} u_{\xi_{j}}^{2} d \xi \sim R^{\sum_{i=1}^{n} \sigma_{i}-\beta} \int_{T}\left|\nabla_{\xi} u\right|^{2} d \xi .
$$

Identity (4.3) implies that $v(\xi)$ is a positive solution of the equation

$$
\sum_{i, j=1}^{n} \frac{\partial}{\partial \xi_{i}}\left(b_{i j}(\xi) \frac{\partial v}{\partial \xi_{j}}\right)=0
$$


from $W_{2}^{1}(T)$ on $T$. In view of (4.5), equation (4.6) is elliptic on $T$ and therefore the Harnack inequality

$$
\underset{\xi \in T^{\prime}}{\operatorname{ess} \sup } v(\xi) \leq C \underset{\xi \in T^{\prime}}{\operatorname{ess} \inf } v(\xi)
$$

is fulfilled for it (see [16]). Here the constant $C=C(n, \mu, \alpha, \beta)$. By the inverse transformation $\xi \rightarrow x$, from estimate (4.7) we derive (4.1).

\section{Lemma on the Increase of Positive Solutions}

Lemma 5.1. Let $D \subset Q_{R}^{0}$ be the domain having limiting points on the surface $\partial Q_{R}^{0}$ and a nonempty intersection with $Q_{R / 4}^{0}$. Let $u \in W_{2, \alpha}^{1}(D)$ be a solution of equation (1.1) in $D, u(x) \leq 0$ on a part of the boundary $\Gamma$ lying strictly inside $Q_{R}^{0}$ in the sense of $W_{2, \alpha}^{1}(D)$. Then there exists a constant $\eta_{0}=\eta_{0}(n, \mu, \alpha, \beta)$ such that for any such function $u(x)$ there holds

$$
\underset{x \in D}{\operatorname{essup}} u(x) \geq\left(1+\eta_{0} R^{\beta-\sum_{j=1}^{n} \sigma_{j}} \operatorname{cap}_{\alpha} H_{R}\right) \underset{x \in D \cap Q_{R / 4}^{0}}{\operatorname{essips}_{x \in D}} u(x),
$$

where the set $H_{R}=Q_{R / 4}^{0} \backslash D$ and $\operatorname{cap}_{\alpha} H_{R}$ is its capacity.

Proof. Denote $M=\sup _{x \in D} u(x), G=Q_{R}^{0} \backslash H_{R}$ and let $U_{H} \in \stackrel{\circ}{W}_{2, \alpha}^{1}\left(Q_{R}^{0}\right)$ be the potential of the set $H_{R}$. Let $\phi \in \operatorname{Lip}_{0}\left(Q_{R}^{0}\right)$ be the function equal to 1 on $H_{R}$. Assume that $z=M\left(1-U_{H}\right)$ in $Q_{R}^{0}$. Then $z$ is a solution of equation (1.1) in $G$, $z(x) \geq 0$, on $H_{R}$. Applying the maximum principle to the functions $z(x), u(z)$ in the domain $D$, we have $z(x) \geq u(x)$ on $\Gamma \cup \partial Q_{R}^{0}$, since $\left.z\right|_{\partial Q_{R}^{0}}=M \geq u(x)$ and, for $x \in \Gamma u(x)=0 \leq z(x)$. Then by virtue of Lemma 2.2 for almost all $x \in D$ we obtain $z(x) \geq u(x)$. Since $\sup _{x \in D \cap Q_{R / 2}^{0}} u(x) \leq \sup _{x \in D \cap Q_{R / 2}^{0}} z(x)$, we have

$$
M \geq M \inf _{x \in D \cap Q_{R / 2}^{0}} U_{H}+\sup _{x \in D \cap Q_{R / 2}^{0}} u(x) .
$$

Let us estimate the expression $\inf _{x \in D \cap Q_{R / 2}^{0}} U_{H}$ from below. To this end, we obtain the uniform estimate (from below) of the numbers $a^{(k)}=\sup _{x \in Q_{R / 2}^{0}} U_{H}^{(k)}$ with sufficiently large $k$ for the corresponding approximating sequence $U_{H}^{(k)}$. Denote the $k$-dependent set $\Gamma_{t}=\left\{x \in Q_{R}^{0}: U_{H}^{(k)}(x)>t\right\}$. Then, by virtue of Lemma 3.4, for an arbitrarily small $\varepsilon>0$ and $a_{1} \in\left(a^{(k)}, a^{(k)}+\varepsilon\right)$ we have

$$
\int_{\partial \Gamma_{1-\varepsilon}} \nabla U_{H}^{(k)} \cdot A N d \sigma=\int_{\partial \Gamma_{a_{1}}} \nabla U_{H}^{(k)} \cdot A N d \sigma+\delta_{k},
$$

where $\delta_{k} \rightarrow 0$ as $k \rightarrow \infty$. Note that $\Gamma_{a_{1}} \subset Q_{R / 2}^{0}, N=\nabla U_{H}^{(k)} /\left|\nabla U_{H}^{(k)}\right|$ on $\partial \Gamma_{1-\varepsilon}$. By virtue of Lemma 3.3, the left-hand side of (5.3) is estimated by the 
expression

$$
\int_{\partial \Gamma_{1-\varepsilon}} \nabla U_{H}^{(k)} \cdot A N d \sigma \geq \frac{1}{1-\varepsilon} \int_{Q_{R}^{0} \backslash \Gamma_{1-\varepsilon}} \nabla U_{H}^{(k)} \cdot A \nabla U_{H}^{(k)} d x+\delta_{k},
$$

which in its turn exceeds

$$
\frac{\mu}{1-\varepsilon} \int_{Q_{R}^{0} \backslash \Gamma_{1-\varepsilon}} \rho(x)^{\alpha} \nabla U_{H}^{(k)} \cdot \nabla U_{H}^{(k)} d x+\delta_{k} \geq \mu(1-\varepsilon) \overline{\operatorname{cap}}_{\alpha} \Gamma_{1-\varepsilon}+\delta_{k} .
$$

The set $H_{R} \subset \Gamma_{1-\varepsilon}$ and therefore the right-hand side of inequality (5.5) is not smaller than $\mu(1-\varepsilon)^{2} \operatorname{cap}_{\alpha} H_{T}$. Then (5.4) implies

$$
\int_{\partial \Gamma_{1-\varepsilon}} \nabla U_{H}^{(k)} \cdot A N d \sigma \geq \mu(1-\varepsilon) \overline{\operatorname{cap}}_{\alpha} H_{R}+\delta_{k}
$$

where $\overline{\operatorname{cap}}_{\alpha} H_{R}$ is a relative capacity $H_{R}$ with respect to $Q_{R}^{0}$.

By virtue of Lemma 3.3, for the right-hand part of (5.3) we obtain

$$
\int_{\partial \Gamma_{a_{1}}} \nabla U_{H}^{(k)} \cdot A N d \sigma \leq \frac{1}{a_{1}} \int_{Q_{R}^{0} \backslash \Gamma_{a_{1}}} \nabla U_{H}^{(k)} \cdot A \nabla U_{H}^{(k)} d x+\delta_{k} .
$$

Let $\varphi_{a_{1}} \in \stackrel{\circ}{W}{ }_{2, \alpha}^{1}\left(Q_{R}^{0}\right)$ be the potential of the set $\bar{\Gamma}_{a_{1}}$ generated by functional (3.6). Then for this potential we have

$$
\int_{Q_{R}^{0}} \rho^{\alpha} \nabla \varphi_{a_{1}} \cdot \nabla \varphi_{a_{1}} d x=\overline{\operatorname{cap}}_{\alpha} \bar{\Gamma}_{a_{1}}
$$

(see (3.7)). Choosing the test function $\varphi=U_{H}^{(k)}-a_{1} \varphi_{a_{1}}$ from equation (1.1), we obtain (since $\varphi \in \stackrel{\circ}{W}{ }_{2, \alpha}^{1}\left(Q_{R}^{0} \backslash \bar{\Gamma}_{a_{1}}\right)$ )

$$
\begin{aligned}
& \int_{Q_{R}^{0} \backslash \Gamma_{a_{1}}} \nabla U_{H}^{(k)} \cdot A \nabla U_{H}^{(k)} d x=a_{1} \int_{Q_{R}^{0} \backslash \Gamma_{a_{1}}} \nabla U_{H}^{(k)} \cdot A \nabla \varphi_{a_{1}} d x+\delta_{k} \\
& \leq a_{1}\left(\int_{Q_{R}^{0}} \nabla U_{H}^{(k)} \cdot A \nabla U_{H}^{(k)} d x\right)^{1 / 2}\left(\int_{Q_{R}^{0}} \nabla \varphi_{a_{1}} \cdot A \nabla \varphi_{a_{1}} d x\right)^{1 / 2}+\delta_{k} .
\end{aligned}
$$

Therefore

$$
\begin{aligned}
\int_{Q_{R}^{0} \backslash \Gamma_{a_{1}}} \nabla U_{H}^{(k)} \cdot A \nabla U_{H}^{(k)} d x & \leq \mu^{-1} a_{1}^{2} \int_{Q_{R}^{0} \backslash \Gamma_{a_{1}}} \nabla \varphi_{a_{1}} \cdot \nabla \varphi_{a_{1}} d x+\delta_{k} \\
& =\mu^{-1} a_{1}^{2} \operatorname{cap}_{\alpha} \bar{\Gamma}_{a_{1}}+\delta_{k} \leq \mu^{-1} a_{1}^{2} \operatorname{cap}_{\alpha} Q_{R / 2}^{0}+\delta_{k},
\end{aligned}
$$

since $\Gamma_{a_{1}} \subset \Gamma_{a^{(k)}} \subset Q_{R / 2}^{0}$ and (5.7) is fulfilled. 
From (5.3), (5.5) , (5.6), (5.8) it follows that

$$
(1-\varepsilon) \mu^{2} \overline{\operatorname{cap}}_{\alpha} H_{R} \leq a_{1} \overline{\operatorname{cap}}_{\alpha} Q_{R / 2}^{0}+\frac{\delta_{k}}{a_{1}}+\delta_{k} .
$$

We replace here $\frac{\delta_{k}}{a_{1}}$ by $\delta_{k}$, since $a_{1} \in\left(a^{(k)}, a^{(k)}+\varepsilon\right)$ and, for sufficiently large $k^{\prime}$ 's, $\alpha^{(k)}$ is close to $\inf _{x \in Q_{R / 2}^{0}} U_{H}$. Hence, taking into account the arbitrariness of $\varepsilon$, we have

$$
a^{(k)} \geq \mu^{2}\left(\frac{\overline{\operatorname{cap}}_{\alpha} H_{R}}{\overline{\operatorname{cap}}_{\alpha} Q_{R / 2}^{0}}\right)+\delta_{k}
$$

It is obvious that

$$
\overline{\operatorname{cap}}_{\alpha} H_{R} \geq \operatorname{cap}_{\alpha} H_{R}
$$

Let $\eta(x)=\prod_{j=1}^{n} \eta_{0}\left(\frac{x_{j}}{R^{\sigma_{j}}}\right), \eta_{0}(t)$ be an infinite times differentiable function on $(-\infty, \infty)$, with a compact support, $0 \leq \eta(t) \leq 1,\left|\eta^{\prime}(t)\right| \leq C_{0} ; C_{0}=C_{0}(n)$, $\eta(t)=1$ for $t \in\left[0, \frac{1}{2}\right], \eta(t)=0$ for $|t| \geq 1$. Then

$$
\overline{\operatorname{cap}}_{\alpha} Q_{R / 2}^{0} \leq \int_{Q_{R}^{0}} \rho^{\alpha} \nabla \eta \cdot \nabla \eta d x
$$

Therefore

$$
\operatorname{cap}_{\alpha} Q_{R / 2}^{0} \leq C_{0} \sum_{j=1}^{n} R^{-2 \sigma_{j}} \int_{Q_{R}^{0}} \rho(x)^{\alpha_{j}} d x \leq C_{0} C_{1} R^{\sum_{i=1}^{n} \sigma_{i}-\beta}
$$

$C_{1}=C_{1}(n, \alpha)$, since for condition (1.3) we have

$$
\int_{Q_{R}^{0}} \rho(x)^{\alpha_{j}} d x \leq C_{1} R^{\sum_{i=1}^{n} \sigma_{i}+\alpha_{j}}
$$

By (5.11), (5.13) and (5.10) we obtain

$$
a^{(k)} \geq C R^{\beta-\sum_{i=1}^{n} \sigma_{i}} \operatorname{cap}_{\alpha} H_{R}+\delta_{k},
$$

where $C=C(n, \mu, \alpha, \beta), \delta_{k} \rightarrow 0$ as $k \rightarrow \infty$. If in estimate (5.15) we assume that $k \rightarrow \infty$ and apply Theorem 4.1 , then we have

$$
\inf _{x \in Q_{R / 1}^{0}} U_{H} \geq \eta_{0} R^{\beta-\sum_{i=1}^{n} \sigma_{i}} \operatorname{cap}_{\alpha} H_{R}
$$

where $\eta_{0}=\eta_{0}(n, \mu, \alpha, \beta)$. Due to (5.16), from (5.2) we obtain (5.1) (keeping in mind that $\sup u \geq \sup u$ and using the maximum principle).

$$
Q_{R / 2}^{0} \quad Q_{R / 4}^{0}
$$




\section{Regularity of a Boundary Point}

Let $D$ be a bounded domain and $f$ a continuous function on $\partial D$. We will consider the Dirichlet problem

$$
L u=0 \quad \text { in } D,\left.\quad u\right|_{\partial D}=f .
$$

Assume that $\phi \in W_{2, \alpha}^{1}(D)$. Using the classical theory of a Hilbert space (or the variational method of $\S 3$ ), we easily prove the existence and uniqueness of a solution of the problem

$$
L u=0 \quad \text { in } \quad D, \quad u-\phi \in \stackrel{\circ}{W}_{2, \alpha}^{1}(D) .
$$

Let us now proceed to constructing a solution of problem (6.1). There exists a sequence of smooth, for example, Lipschitz functions $f_{k}$ approximating $f$ uniformly on $\partial D$. There also exists an extension $\phi_{k}$ of functions $f_{k}$ from $\partial D$ to all $\bar{D}$ with the preservation of its Lipschitz property (see $\left[15\right.$, p. 206]). Let $u_{k}$ be a solution of problem (6.2) for $\phi=\phi_{k}$. Then $u_{k}$ is a fundamental sequence in the uniform metric. Indeed, according to the maximum principle, we have

$$
\underset{\bar{D}}{\operatorname{ess} \sup }\left|u_{k}-u_{m}\right| \leq \sup _{\partial D}\left|u_{k}-u_{m}\right|=\sup _{\partial D}\left|f_{k}-f_{m}\right| \rightarrow 0 \quad \text { as } \quad k, m \rightarrow \infty .
$$

Therefore the sequence $u_{k} \rightarrow u_{f}$ uniformly in $\bar{D}$ to some function $u_{f}(x)$. The function $u_{f}$ is a solution of equation (1.1) in any strictly internal domain $D^{\prime} \subset$ $D$. Indeed, let $\xi \in \operatorname{Lip}_{0}(D)$ be equal to 1 in $D^{\prime}$ and to 0 outside $D$. Then we have $\int_{D} \nabla u_{k} \cdot A \nabla\left(u_{k} \xi^{2}\right) d x=0$, whence it follows that $\int_{D} \nabla u_{k} \cdot A \nabla u_{k} \xi^{2} d x \leq$ $4 \int_{D} \nabla \xi \cdot A \nabla \xi d x$. Therefore

$$
\int_{D^{\prime}} \rho^{\alpha} \nabla u_{k} \cdot \nabla u_{k} d x \leq \mu^{-1} \int_{D^{\prime}} \nabla u_{k} \cdot A \nabla u_{k} d x \leq C\left(D^{\prime}\right),
$$

where $C\left(D^{\prime}\right)$ does not depend on $k$. Then $u_{k_{n}} \rightarrow v$ weakly in $W_{2, \alpha}^{1}\left(D^{\prime}\right)$ for some subsequence $u_{k_{n}}$. Since, in addition, $u_{k} \rightarrow u_{f}$ uniformly, we have $v=u_{f}$. Thus the function $u_{f}$, which does not depend on the approximation technique, is a solution of equation (1.1) in $D^{\prime}$. The function $u_{f}(x)$ is called a generalized solution of problem (6.1).

Definition 6.1. A point $x_{0} \in \partial D$ is called regular if a generalized solution $u_{f}(x)$ of problem (6.1) is continuous at $x_{0}$ for any continuous function $f$ on $\partial D$.

The behavior of the solution of the Dirichlet problem for equation (1.1) near a nonregular boundary point depends on the domain geometry in an immediate proximity of this point. Since for the boundary points $x_{0} \neq 0$ equation (1.1) has no degeneration, the regularity criterion for these points coincides with Wiener's criterion for the Laplace equation (see [3]). Hence we show interest in boundary point 0 . The main result of this work is 
Theorem 6.1. Let $D \subset E_{n}$ be a bounded domain, $0 \in \partial D$. Then for boundary point 0 to be regular for equation (1.1) it is necessary and sufficient that

$$
\int_{0} R^{\beta-\sum_{i=1}^{n} \sigma_{i}} \operatorname{cap}_{\alpha} H_{R} \frac{d R}{R}=\infty,
$$

or, which is the same,

$$
\sum_{m=1}^{n} \gamma\left(4^{-m}\right)=\infty
$$

where the set $H_{R}=Q_{R}^{0} \backslash D$ and $\operatorname{cap}_{\alpha} H_{R}$ is its capacity, $\gamma(t)=t^{\beta-\sum_{i=1}^{n} \sigma_{i}} \operatorname{cap}_{\alpha} H_{t}$.

Proof. Sufficiency. Because of the continuity of the function $f$, for any $\varepsilon>0$, there exists $0<\delta_{1}<1$ such that $|f(x)-f(0)|<\frac{\varepsilon}{2}$ for $|x|<\delta_{1}, x \in D$. We have $f_{k} \in \operatorname{Lip}(\partial D)$ for which $\left|f_{k}-f\right|<\frac{\varepsilon}{2}$ when $x \in \partial D$. Let $u_{k}$ be a solution of the problem

$$
L u_{k}=0 \quad \text { in } \quad D, \quad u_{k}-\phi_{k} \in \stackrel{\circ}{W}_{2, \alpha}^{1}(D),
$$

where $\phi_{k} \in \operatorname{Lip}(\bar{D})$ is an extension of $f_{k}$ with preservation of its Lipshitz property to all $\bar{D}$ (for the existence of such an extension see [15, Ch. VI, p. 206]). The function $z=u_{k}-f\left(x_{0}\right)-\varepsilon$ is also a solution of the equation $L z=0$ in $D$ for any $x \in\left\{|x|<\delta_{1}\right\} \cap D$. The function $z(x)$ is continuous in $D$. Denote $D^{\prime}=\{x \in D: z(x)>0\}, \sigma_{0}=\min _{1 \leq k \leq n}\left\{\sigma_{k}\right\}$. Let $m_{0} \in N, 4^{-m_{0} \sigma_{0}}<\delta_{1}$. Then $Q_{4^{-m_{0}}}^{0} \subset\left\{|x|<\delta_{1}\right\}$ and applying Lemma 5.1 to $Q_{4^{-m+1}}^{0}$ and $Q_{4^{-m}}^{0}$ and assuming that $m=m_{0}+1, m_{0}+2, \ldots, l$, we have

$$
M_{m-1} \geq\left(1+\eta_{0} \gamma_{m}\right) M_{m},
$$

where $\gamma_{m}=\gamma\left(4^{-m}\right), M_{m}=\sup _{Q_{4-m}^{0}} z$.

Applying repeatedly estimate (6.6) we obtain

$$
\begin{aligned}
M_{m_{0}} & \geq \prod_{j=m_{0}}^{l}\left(1+\eta_{0} \gamma_{j}\right) M_{l} \geq M_{l} \exp \left(\sum_{j=m_{0}}^{l} \ln \left(1+\eta_{0} \gamma_{j}\right)\right) \\
& \geq M_{l} \exp \left(\eta_{0} C_{0} \sum_{j=m_{0}}^{l} \gamma_{j}\right),
\end{aligned}
$$

where $C_{0}=C_{0}(n)$. Hence

$$
M_{l} \leq M_{m_{0}} \exp \left(-\eta_{0} C_{0} \sum_{j=m_{0}}^{l} \gamma_{j}\right) .
$$

An analogous estimate holds for the function $z_{1}=f\left(x_{0}\right)+\varepsilon-u_{k}$, too:

$$
\mu_{l} \leq \mu_{m_{0}} \exp \left(-\eta_{0} C_{0} \sum_{j=m_{0}}^{l} \gamma_{j}\right)
$$


where $\mu_{m}=\sup _{x \in D \cap Q_{4^{-}}^{0}} z_{1}(x)$. From (6.7) and (6.8) we obtain the estimate for $\left|u_{k}(x)-f(0)\right|$. Since $u_{k} \rightarrow u_{f}$ uniformly in $\bar{D}$, we have

$$
\sup _{D \cap Q_{4^{-l}}^{0}}\left|u_{f}(x)-f(0)\right| \leq \varepsilon+2 \max _{\partial D \cap Q_{4^{-m}}^{0}}|f(x)| \exp \left(-\eta_{0} C_{0} \sum_{j=m_{0}}^{l} \gamma_{j}\right) .
$$

Choosing $l=l(\varepsilon) \in N$ so that the second summand is smaller than $\varepsilon$ for $l>l(\varepsilon)$, we obtain $\left|u_{k}(x)-f(0)\right|<2 \varepsilon$ for $x \in Q_{\delta}^{0} \cap D\left(\delta=\min \left(4^{-l(\varepsilon)}, \delta_{1}^{1 / \sigma_{0}}\right)\right)$ and therefore for $|x|<\delta^{\sigma^{0}}\left(\sigma^{0}=\max _{1 \leq i \leq n} \sigma_{i}\right)$, too. Applying an analogous reasoning to regular boundary point 0 , the continuity modulus of the solution $u_{f}(x)$ is estimated through the continuity modulus of the function $f$ and the sum of series (6.4).

Let $|f(x)-f(0)|<\theta(|x|)$, where $\theta(x) \downarrow 0$ is the continuity modulus. Then

$$
\begin{array}{r}
\left|u_{f}(x)-f(0)\right| \leq 2 \max _{\partial D}|f| \exp \left(-C_{0} \int_{\rho(x)}^{\delta_{1}^{1 / \sigma^{0}}} \tau^{\beta-\sum_{i=1}^{n} \sigma_{i}} \operatorname{cap}_{\alpha} H_{\tau} \frac{d \tau}{\tau}\right)+\theta(\delta) \\
\text { for } \rho(x) \leq \delta_{1}^{1 / \sigma^{0}} .
\end{array}
$$

Such estimates for equation (1.1) were obtained for the first time in [17].

To prove the necessity we need the following

Proposition 6.1. Let $H \subset Q_{R}^{0} \backslash Q_{R / 4}^{0}$ be the closed set, and $v_{H} \in \stackrel{\circ}{W_{2, \alpha}^{1}}\left(E_{n}\right)$ be the potential of the set $H$. Then

$$
\sup _{x \in Q_{R / 8}^{0}} v_{H} \leq C R^{\beta-\sum_{j=1}^{n} \sigma_{j}} \operatorname{cap}_{\alpha} H, \quad C=C(n, \mu, \beta, \alpha) .
$$

To show estimate (6.11) for sufficiently large $k \in N$, we obtain the uniform estimate for the approximating sequence $v_{H}^{(k)}(x)$.

Let $t \in(0,1), \Gamma_{t}=\left\{x \in E_{n}: v_{H}^{(k)}(x)>t\right\}, b^{(k)}=\inf _{x \in Q_{R / 8}^{0}} v_{H}^{(k)}(x)$. Then $Q_{R / 8}^{0} \subset \Gamma_{b^{(k)}}$. Using Lemmas 3.3 and 3.4, for an arbitrary small $\varepsilon>0$ and $b_{1} \in\left(b^{(k)}-\varepsilon, b^{(k)}\right)$ we obtain

$$
\begin{aligned}
& \int_{E_{n} \backslash \bar{\Gamma}_{b_{1}}} \nabla v_{H}^{(k)} \cdot A \nabla v_{H}^{(k)} d x \leq b_{1} \int_{\partial \Gamma_{b_{1}}} \nabla v_{H} \cdot A N d \sigma+\delta_{k} \\
& \quad \leq \delta_{k}+b_{1} \int_{\partial \Gamma_{1-\varepsilon}} \nabla v_{H}^{(k)} \cdot A N d x \leq \frac{b_{1}}{1-\varepsilon} \int_{E_{n} \backslash \Gamma_{1-\varepsilon}} \nabla v_{H}^{(k)} \cdot A \nabla v_{H}^{(k)} d x+\delta_{k},
\end{aligned}
$$


where $\delta_{k} \rightarrow 0$ as $k \rightarrow \infty$. If $Q_{R / 8}^{0} \subset \Gamma_{b_{1}}$, then we have

$$
\begin{aligned}
\int_{E_{n} \backslash \bar{\Gamma}_{b_{1}}} \nabla v_{H}^{(k)} \cdot A \nabla v_{H}^{(k)} d x & \geq \mu \int_{E_{n} \backslash \Gamma_{b_{1}}} \rho^{\alpha} \nabla v_{H}^{(k)} \cdot \nabla v_{H}^{(k)} d x \\
& \geq \mu b_{1}^{2} \operatorname{cap}_{\alpha} \bar{\Gamma}_{b_{1}} \geq \mu b_{1}^{2} \operatorname{cap}_{\alpha} Q_{R / 8}^{0} .
\end{aligned}
$$

Let us estimate $\operatorname{cap}_{\alpha} Q_{R / 8}^{0}$ from below. Clearly, $\operatorname{cap}_{\alpha} Q_{R / 8}^{0} \geq \operatorname{cap}_{\alpha}\left(Q_{R / 8}^{0}\right)$ $\left.Q_{R / 16}^{0}\right)$. Denote $K=Q_{R / 8}^{0} \backslash Q_{R / 16}^{0}$. Then $\operatorname{cap}_{\alpha} K=\inf \int_{E_{n}} \rho^{\alpha}(x) \nabla \varphi \cdot \nabla \varphi d x$, where the lower bound is taken over all $\varphi \in \operatorname{Lip}_{0}\left(E_{n}\right)$ equal to 1 on $K$. It is obvious that

$$
\operatorname{cap}_{\alpha} K \sim \inf _{\{\varphi\}} \sum_{j=1}^{n} R^{\alpha_{j}} \int_{E_{n}} \varphi_{x_{j}}^{2} d x
$$

If we make the change $x \rightarrow \xi$ by the formula $x=R^{\sigma} \xi=\left(R^{\sigma_{1}} \xi_{1}, R^{\sigma_{2}} \xi_{2}, \ldots, R^{\sigma_{n}} \xi_{n}\right)$ in integral (6.14), then $K$ transforms to $P=Q_{1 / 8}^{0} \backslash Q_{1 / 16}^{0}$ and we have

$$
\sum_{j=1}^{n} R^{\alpha_{j}} \int_{E_{n}} \varphi_{x_{j}}^{2} d x=R^{\sum_{i=1}^{n} \sigma_{i}-\beta} \int_{E_{n}}|\nabla \psi|^{2} d \xi
$$

where $\psi(\xi)=\varphi(x(\xi))$. Minimizing the right-hand side of (6.15) over all admissible functions $\psi$, we obtain

$$
\sum_{j=1}^{n} R^{\alpha_{j}} \int_{E_{n}} \varphi_{x_{j}}^{2} d x \geq C_{1} R^{\sum_{i=1}^{n} \sigma_{i}-\beta}
$$

Here the value $C_{1}=C_{1}(n, \mu, \alpha, \beta)$ is equal to the Wiener capacity of the compactum $P$ which exceeds some constant $C(n, \mu, \alpha, \beta)$. From (6.14), (6.15) and (6.16) we find

$$
\operatorname{cap}_{\alpha} Q_{R / 8}^{0} \geq C R^{\sum_{j=1}^{n} \sigma_{j}-\beta}, \quad C=C(n, \mu, \alpha, \beta) .
$$

Taking into account (6.13) and (6.17), we obtain

$$
\int_{E_{n} \backslash \Gamma_{b_{1}}} \nabla v_{H}^{(k)} \cdot A \nabla v_{H}^{(k)} d x \geq C b_{1}^{2} R^{\sum_{j=1}^{n} \sigma_{j}-\beta} .
$$

Since $H \subset \Gamma_{1-\varepsilon}$, we have

$$
\int_{E_{n} \backslash \Gamma_{1-\varepsilon}} \nabla v_{H}^{(k)} \cdot A \nabla v_{H}^{(k)} d x \leq \int_{E_{n} \backslash H} \nabla v_{H}^{(k)} \cdot A \nabla v_{H}^{(k)} d x \leq \mu^{-1} \operatorname{cap}_{\alpha} H .
$$

From estimates (6.18), (6.19) and (6.12) we obtain the estimate for $b_{1}$, whence by virtue of the arbitrariness of $\varepsilon$ we have

$$
b^{(k)} \leq C R^{\beta-\sum_{j=1}^{n} \sigma_{j}} \operatorname{cap}_{\alpha} H .
$$


Passing to the limit as $k \rightarrow \infty$, from (6.20) we obtain the estimate for $\inf _{x \in Q_{R / 8}^{0}} v_{H}(x)$ and thus, applying Theorem 4.1, we have

$$
\sup _{x \in Q_{R / 8}^{0}} v_{H}(x) \leq C R^{\beta-\sum_{j=1}^{n} \sigma_{j}} \operatorname{cap}_{\alpha} H
$$

$C=C(n, \mu, \alpha, \beta)$. Estimate (6.11) is proved.

Necessity. Assume that condition (6.4) is not fulfilled. Let $U(x)$ be the potential of the set $Q_{4^{-m_{0}}}^{0} \backslash D$, the integer $m_{0} \in N$ will be chosen later. We will show that for any $\delta>0$ there exists a point $x^{\prime} \in Q_{\delta}^{0}, x^{\prime} \neq 0, x^{\prime} \in D$, for which $U\left(x^{\prime}\right)<\frac{1}{2}$. Since series (6.4) is convergent, there is an integer $m_{1}$ for which $4^{-m_{1}}<\delta$

$$
\gamma_{m_{1}-1} \leq \frac{\mu^{2}}{8}
$$

Let us denote by $U_{0}(x) \in \stackrel{\circ}{W_{2, \alpha}^{1}}\left(E_{n}\right)$ the potential of the set $G_{m_{1}-1}=Q_{4^{-m_{1}+1}}^{0} \backslash$ $D$ and show that

$$
\inf _{x \in Q_{4^{-m_{1}-1}}^{0}} U_{0}<\frac{1}{4} .
$$

We again pass over to the approximating sequence $U_{0}^{(k)}(x)$. For this, we denote $a^{(k)}=\inf _{x \in Q_{4^{-} m_{1}}^{0}} U_{0}^{(k)}(x), \Gamma_{t}=\left\{x \in E_{n}: U_{0}^{(k)}(x)>t\right\}, t \in(0,1)$. Then, taking into account that $Q_{4^{-m}}^{0} \subset \Gamma_{a^{(k)}}$ and using Lemmas 3.3, 3.4, for arbitrarily small $\varepsilon>0$ and $a_{1} \in\left(a^{(k)}-\varepsilon, a^{(k)}\right)$ we have

$$
\begin{aligned}
\mu C_{1} 4^{-m q} a_{1}^{2} \leq \mu a_{1}^{2} \operatorname{cap}_{\alpha} \bar{\Gamma}_{a_{1}} \leq \mu \int_{E_{n} \backslash \Gamma_{a_{1}}} \rho^{\alpha} \nabla U_{0}^{(k)} \cdot \nabla U_{0}^{(k)} d x \\
\quad \leq \int_{E_{n} \backslash \Gamma_{a_{1}}} \nabla U_{0}^{(k)} \cdot A \nabla U_{0}^{(k)} d x=a_{1} \int_{\partial \Gamma_{a_{1}}} \nabla U_{0}^{(k)} \cdot A N d \sigma+\delta_{k} \\
\quad=a_{1} \int_{\partial \Gamma_{1-\varepsilon}} \nabla U_{0}^{(k)} \cdot A N d \sigma=\frac{a_{1}}{1-\varepsilon} \int_{E_{n} \backslash \Gamma_{a_{1}}} \nabla U_{0}^{(k)} \cdot A \nabla U_{0}^{(k)} d x+\delta_{k} .
\end{aligned}
$$

Let $\psi \in \stackrel{\circ}{W} \stackrel{1}{2, \alpha}^{1}\left(E_{n}\right)$ be the potential of the set $\Gamma_{1-\varepsilon}$ generated by functional (3.6). Then $\varphi=u_{0}^{(k)}-(1-\varepsilon) \psi \in \stackrel{\circ}{W_{2, \alpha}^{1}}\left(E_{n} \backslash \Gamma_{1-\varepsilon}\right)$ is the test function for (1.1) in $E_{n} \backslash \Gamma_{1-\varepsilon}$ and therefore

$$
\int_{E_{n} \backslash \Gamma_{1-\varepsilon}} \nabla U_{0}^{(k)} \cdot A \nabla \varphi d x=\delta_{k} \rightarrow 0
$$

or

$$
\int_{E_{n} \backslash \Gamma_{1-\varepsilon}} \nabla U_{0}^{(k)} \cdot A \nabla U_{0}^{(k)} d x=(1-\varepsilon) \int_{E_{n} \backslash \Gamma_{1-\varepsilon}} \nabla U_{0}^{(k)} \cdot A \nabla \psi d x+\delta_{k},
$$


whence we find

$$
\begin{aligned}
\int_{E_{n} \backslash \Gamma_{1-\varepsilon}} \nabla u_{0}^{(k)} \cdot A \nabla u_{0}^{(k)} d x & \leq(1-\varepsilon)^{2} \int_{E_{n} \backslash \Gamma_{1-\varepsilon}} \nabla \psi \cdot A \nabla \psi d x+\delta_{k} \\
& \leq \delta_{k}+\mu^{-1}(1-\varepsilon)^{2} \int_{E_{n} \backslash \Gamma_{1-\varepsilon}} \rho^{\alpha}(x) \nabla \psi \cdot \nabla \psi d x \\
& =\mu^{-1}(1-\varepsilon)^{2} \operatorname{cap}_{\alpha} \bar{\Gamma}_{1-\varepsilon}+\delta_{k} .
\end{aligned}
$$

Now, since $\varepsilon>0$ is arbitrary, we conclude that the right-hand part of (6.24) does not exceed

$$
\frac{3}{2} \mu^{-1} \operatorname{cap}_{\alpha} G_{m_{1}-1}
$$

Therefore (6.23) implies that

$$
a^{(k)} \leq \frac{3}{2} \mu^{-2} 4^{m q} \operatorname{cap}_{\alpha} G_{m_{1}-1}+\delta_{k}, \quad q=\sum_{i=1}^{n} \sigma_{i}-\beta .
$$

Making $k \rightarrow \infty$, from the latter inequality we obtain

$$
\inf _{x \in Q_{4^{-m_{1}}}^{0}} U_{0}(x)<\frac{3}{16} .
$$

Then there exists a point $x^{\prime} \in Q_{\delta}^{0}$ such that $U_{0}\left(x^{\prime}\right)<\frac{1}{4}$. We put

$$
U=\sum_{m=m_{0}}^{m_{1}-2} U_{m}+U_{0}
$$

where $U_{m} \in \stackrel{\circ}{W}_{2, \alpha}^{1}\left(E_{n}\right)$ is the potential of the set $K_{m}=\left(\bar{Q}_{4^{-m}}^{0} \backslash Q_{4^{-m-1}}^{0}\right) \backslash D$. Now, applying estimate (6.9) of Proposition 6.1 to this potential and assuming $m=m_{0}+1, m_{0}+2, \ldots, m_{1}-2$, we obtain

$$
U_{m}\left(x^{\prime}\right) \leq \sup _{x \in Q_{4^{-m-2}}^{0}} U_{m}(x) \leq C 4^{m q} \operatorname{cap}_{\alpha} K_{m}, \quad C=C(n, \mu, \alpha, \beta) .
$$

Therefore $U\left(x^{\prime}\right) \leq \frac{1}{4}+C \sum_{m=m_{0}}^{m_{1}-2} \gamma_{m}$.

Let us now choose $m_{0}$ so large that the latter sum be $<\frac{1}{4}$. Then $U\left(x^{\prime}\right)<\frac{1}{2}$ and the necessity is proved.

The approach to the construction of the potential $U$ is analogous to that presented in $[18$, p. 43], while the proof of the sufficiency begins as described in $[19$, p. 50].

In the case of nondegenerated elliptic equations of second order we know the sufficient conditions for a boundary point which are of obvious geometric character, say, when the boundary points may have contact with the cone or funnel vertex lying outside the domain [18, pp. 44, 45]. In Proposition 6.2 below we give an analogous sufficient condition for the regularity of the boundary point at which the equation degenerates. 
Condition K. Let $g \subset Q_{h_{0}}^{0}$ be some set of positive Wiener capacity ( $\operatorname{cap}_{\alpha} g \neq$ $0, \alpha=0), 0 \in \partial g$, where $h_{0}$ is some integer.

Assume that for every $t \in\left(0, h_{0}\right)$, the transformation

$$
y=\left(h_{0} / t\right)^{\sigma} x
$$

brings the set $Q_{t}^{0} \backslash D$ to $g$. Then we say that the domain $D$ satisfies the condition $\mathrm{K}$.

Proposition 6.2. Let $D \subset E_{n}, 0 \in \partial D$. If the domain $D$ satisfies the condition $K$. then the point 0 is regular.

Proof. It is sufficient to apply Theorem 6.1, i.e. to show the divergence of the integral in condition (6.3).

It is not difficult to show the estimate

$$
\operatorname{cap}_{\alpha}\left(Q_{t}^{0} \backslash D\right) \geq C_{1} t^{\sum_{k=1}^{n} \sigma_{k}-\beta}
$$

where $C_{1}$ is the Wiener capacity of the set $g$.

Indeed, let $u(x)$ be a test function for $Q_{t}^{0} \backslash D$, i.e. $v \in C_{0}^{\infty}\left(E_{n}\right), v(x) \geq 1$ on $Q_{t}^{0} \backslash D$. Then according to the condition $\mathrm{K}$ the change of the variables $x=\left(t / h_{0}\right)^{\sigma} y$ transforms the set $Q_{t}^{0} \backslash D$ to $g$. Therefore

$$
\int_{E_{n}} \rho^{\alpha}(x) \nabla v \cdot A \nabla v d x=t \sum_{k=1}^{n} \sigma_{k}-\beta \int_{E_{n}}\left(\nabla_{y} \widetilde{v}\right)^{2} d y,
$$

where $\widetilde{v}(y)=v\left(\left(t / h_{0}\right)^{\sigma} y\right)$. It is not difficult to verify that the function $\widetilde{v}$ in (6.27) is the test function for $g$, i.e. $\left.\widetilde{v}\right|_{g} \geq 1, \widetilde{v} \in C_{0}^{\infty}\left(E_{n}\right)$, whence follows estimate (6.26). Using (6.26) in integral (6.3), we obtain its divergence.

The proposition is proved.

Let the set $K_{1} \equiv K\left(h_{0}\right) \subset Q_{h_{0}}^{0}, 0 \in \partial K_{1}, \operatorname{cap}_{\alpha} K_{1} \neq 0, t \in\left(0, h_{0}\right)$, be such that the transformation

$$
x=\left(t / h_{0}\right)^{\sigma} y
$$

brings this set to the part lying in the ball $Q_{t}^{0}$. We call such a set $K_{1}$ a metric cone with vertex at 0 .

Proposition 6.3. If $0 \in \partial D$ and the point 0 can be contacted outside the domain by the vertex of some metric cone $K_{1}$, then the point is regular.

\section{ACKNOWLEDGEMENT}

The authors express their gratitude to the referee for his helpful comments and remarks that contributed to the elimination of some inaccuracies in the original version of the paper. 


\section{REFERENCES}

1. N. Wiener, The Dirichlet problem. Journ. of Math. Massachussetts 3(1924), 127-146.

2. M. V. Keldysh, On the solubility and the stability of Dirichlet's problem. (Russian) Uspekhi Mat. Nauk 8(1941), 171-231.

3. W. Littman, G. Stampacchia, and H. F. Weiberger, Regular points for elliptic equations with discontinuous coefficients. Ann. Scuola Norm. Sup. Pisa (3) 17(1963), 43-77; Russian transl.: Matematika 9(1966), No. 2, 72-97.

4. V. G. MAZ'YA, The behavior near the boundary of the solution of the Dirichlet problem for an elliptic equation of the second order in divergence form. (Russian) Mat. Zametki 2(1967), 209-220.

5. N. V. KRYLOV, The first boundary value problem for elliptic equations of second order. (Russian) Differencial'nye Uravnenija 3(1967), 315-326.

6. A. A. Novruzov, The regularity of boundary points for an elliptic equation with discontinuous coefficients. (Russian) Vestnik Moskov. Univ. Ser. I Mat. Meh. 26(1971), No. $6,18-25$.

7. Yu. A. Alkhutov, On the regularity of boundary points with respect to the Dirichlet problem for second-order elliptic equations. (Russian) Mat. Zametki 30(1981), No. 3, 333-342.

8. E. B. Fabes, D. Jerison, and C. Kenig, The Wiener test for degenerate elliptic equations. Ann. Inst. Fourier (Grenoble) 32(1982), No. 3, vi, 151-182.

9. I. T. Mamedov and S. T. Guseynov, Interior a priori estimate of the Hölder's norm for the solutions of non-uniformly degenerate second order elliptic equations. Trans. Acad. Sci. Azerb. Ser. Phys.-Tech. Math. Sci. 19(1999), No. 1-2, Math. Mech., 108-121.

10. I. T. MAmedov, On the interior smoothness of solutions of second order elliptic equations with nonuniform power degeneration. (Russian) Trudy IMM AN Azerb. III(XI)(1995), $8-22$.

11. S. T. Guseynov, Harnack type inequality for solution of nonuniformly degenerate equations of second order. (Russian) Dokl. Acad. Nauk Azerb. LV(1999), No. 3-4, 3-11.

12. O. A. Ladyzhenskaya and N. N. Ural'tseva, Linear and quasilinear elliptic equations. (Translated from the Russian) Academic Press, New York-London, 1968; Russian original: Nauka, Moscow, 1967.

13. G. Stampacchia, Le probleme de Dirichlet pour les equations elliptiques du second ordre a coefficients discontinus. Ann. Inst. Fourier (Grenoble) 15(1965), fasc. 1, 189-258.

14. V. G. MaZ'YA, Sobolev spaces. (Russian) Leningrad. Univ., Leningrad, 1985; English transl.: Series in Soviet Mathematics. Springer-Verlag, Berlin, 1985.

15. I. M. Stein, Singular integral and differential properties of functions. (Translated into Russian) Mir, Moscow, 1973.

16. J. Moser, On Harnack's theorem for elliptic differential equations. Comm. Pure Appl. Math. 14(1961), 577-591.

17. V. G. MAZ'YA, On the modulus of continuity of a solution of the Dirichlet problem near an irregular boundary. (Russian) Problems Math. Anal. Boundary Value Problems Integr. Equations (Russian) 45-58; Izdat. Leningrad. Univ., Leningrad, 1966.

18. E. M. Landis, Second order equations of elliptic and parabolic type. (Russian) Nauka, Moscow, 1971.

19. V. A. Kondrat'Ev and E. M. Landis, Qualitative theory of second-order linear partial differential equations. (Russian) Partial differential equations, 3 (Russian), 99-215, 220, Itogi Nauki i Tekhniki, Akad. Nauk SSSR, Vsesoyuz. Inst. Nauchn. i Tekhn. Inform., Moscow, 1988. 
(Received 13.04.2006; revised 24.11.2006)

\author{
Authors' address: \\ Institute of Mathematics and Mechanics \\ of the National Academy of Sciences of Azerbaijan \\ 9, F. Agayev St., Az 1141, Baku \\ Azerbaijan \\ E-mail: farman-m@mail.ru
}

